\title{
Effects of established BMI-associated loci on obesity-related traits in a French representative population sample
}

\author{
Louisa Goumidi, Dominique Cottel, Jean Dallongeville, Philippe Amouyel and Aline Meirhaeghe*
}

\begin{abstract}
Background: Genome-wide association studies have identified variants associated with obesity-related traits, such as the body mass index (BMI). We sought to determine how the combination of 31 validated, BMI-associated loci contributes to obesity- and diabetes-related traits in a French population sample. The MONA LISA Lille study (1578 participants, aged 35-74) constitutes a representative sample of the population living in Lille (northern France). Genetic variants were considered both individually and combined into a genetic predisposition score (GPS).

Results: Individually, 25 of 31 SNPs showed directionally consistent effects on BMI. Four loci (FTO, FANCL, MTIF3 and NUDT3) reached nominal significance $(p \leq 0.05)$ for their association with anthropometric traits. When considering the combined effect of the 31 SNPs, each additional risk allele of the GPS was significantly associated with an increment in the mean $[95 \% \mathrm{Cl}] \mathrm{BMI}$ of $0.13[0.07-0.20] \mathrm{kg} / \mathrm{m}^{2}\left(p=6.3 \times 10^{-5}\right)$ and a $3 \%$ increase in the risk of obesity $(p=0.047)$. The GPS explained $1 \%$ of the variance in the BMI. Furthermore, the GPS was associated with higher fasting glycaemia $(p=0.04)$, insulinaemia $(p=0.008)$, HbA1c levels $(p=0.01)$ and HOMA-IR scores $(p=0.0003)$ and a greater risk of type 2 diabetes (OR [95\% CI] $=1.06[1.00-1.11], p=0.03)$. However, these associations were no longer statistically significant after adjustment for BMI.
\end{abstract}

Conclusion: Our results show that the GPS was associated with a higher BMI and an insulin-resistant state (mediated by BMI) in a population in northern France.

Keywords: Genetic predisposition score, Polymorphism, BMI, Obesity, General population

\section{Background}

According to the World Health Organization (WHO)'s criterion for obesity (body mass index (BMI) $\geq 30 \mathrm{~kg} / \mathrm{m}^{2}$ ), up to $15 \%$ of the adults in Europe are obese [1]. The prevalence of obesity has more or less doubled since 1980 [2]. Obesity is a serious public health issue worldwide. Indeed, there is a well-documented relationship between a high BMI on one hand and mortality and morbidity due to chronic diseases (such as cardiovascular disease, certain cancers, type 2 diabetes (T2D) and osteoarthritis) on the other [3]. Accordingly, the WHO has declared obesity to be a global epidemic that affects both industrialized and non-industrialized countries [4].

\footnotetext{
* Correspondence: Aline.Meirhaeghe@pasteur-lille.fr

INSERM, U744; Institut Pasteur de Lille; Université Lille Nord de France, 1 rue du Pr. Calmette, BP 245, Lille Cedex F-59019, France
}

Body fat mass is influenced by the combination of genetic factors and lifestyle factors (such as diet and physical activity). Family and twin studies have shown that genetic factors account for $40-70 \%$ of the population variation in BMI $[5,6]$; this may explain why people are not all equally affected by obesity in an obesogenic environment [7].

Genome-wide association studies (GWASs) have sought to elucidate the genetic basis of obesity and its related traits. To date, 32 genetic loci have been unequivocally associated with BMI [8]. Several studies have replicated these associations and have taken account of the combined impact of these GWAS-validated loci when considering BMI and other obesity-related phenotypes $[8,9]$. The objective of the present study was to replicate the combined effects of the established BMI-associated loci on BMI, body fat percentage, waist circumference, waist-to-hip ratio (WHR) and obesity risk in a representative sample of the general population in northern France $(n=1578)$. Furthermore, the high 
observed burden of obesity-related co-morbidities (such as insulin resistance and T2D) prompted us to test the impact of the BMI-associated loci on glucose-related traits and the risk of T2D.

\section{Results}

Characteristics of the MONA LISA Lille study

The characteristics of the study participants are summarized in Additional file 1: Table S1. Of the 1578 individuals, $37.3 \%$ were overweight, $22.4 \%$ were obese, and $9.2 \%$ had T2D. We selected 32 single nucleotide polymorphisms (SNPs) in or near the genes listed hereafter and that are known to be associated with BMI: FTO, MC4R, TMEM18, GNPDA2, BDNF, NEGR1, SH2B1, ETV5, MTCH2, KCTD15, TFAP2B, NRXN3, FAIM2, SEC16B, RBJ-ADCY3-POMC, GPRC5B, MAP2K5-LBXCOR1, QP CTL-GIPR, TNNI3K, SLC39A8, FLJ35779-HMGCR, LR RN6C, TMEM160, FANCL, CADM2, PRKD1, LRP1B, PTBP2, MTIF3-GTF3A, RPL27A-TUB, NUDT3-HMGA1 and ZNF608 [8]. Genotyping of the ZNF608 rs4836133 SNP failed and so the remaining 31 SNPs were investigated further.

\section{Single-variant analyses}

All SNPs conformed to Hardy-Weinberg equilibrium (Additional file 1: Table S2). As the sample size was relatively small and the statistical power limited, no SNPs were significantly associated with any of the anthropometric parameters. Only the FTO rs9939609 and FANCL rs887912 SNPs were nominally associated with BMI $(\beta \pm$ $\mathrm{SE}=0.49 \pm 0.19 \mathrm{~kg} / \mathrm{m}^{2}, \quad p=0.008$ and $\beta \pm \mathrm{SE}=0.54 \pm$ $0.19 \mathrm{~kg} / \mathrm{m}^{2}, p=0.005$, respectively). Of the 31 tested SNPs, 25 were directionally consistent with the results reported in the original GWAS on BMI (Additional file 1: Table S3). This number was higher than that expected by chance $(p=0.0003$ in a binomial test). Some SNPs were nominally associated with continuous anthropometric traits other than BMI (such as body fat percentage and hip and waist circumferences). The FTO rs9939609 and FANCL rs887912 SNPs were nominally associated with body fat percentage $(\beta \pm \mathrm{SE}=0.53 \pm 0.27 \%, p=0.05$ and $\beta \pm \mathrm{SE}=0.69 \pm 0.28 \%$, $p=0.01$, respectively). We also observed nominal associations between the FANCL rs887912 SNP and waist and hip circumferences $(\beta \pm \mathrm{SE}=1.03 \pm 0.50 \mathrm{~cm}, p=0.04$ and $\beta \pm$ $\mathrm{SE}=0.89 \pm 0.39 \mathrm{~cm}, p=0.02$, respectively), between the MTIF3 rs4771122 SNP and hip circumference $(\beta \pm \mathrm{SE}=0.84 \pm 0.42 \mathrm{~cm}, p=0.04)$ and between the NUDT3 rs206936 SNP and WHR $(\beta \pm \mathrm{SE}=-0.007 \pm$ $0.003, p=0.01)$.

The genetic predisposition score, BMI and the obesity risk The 31 SNPs were used to calculate a genetic predisposition score (GPS), which was normally distributed (mean: $27.7 \pm 3.7$ alleles; range: 13.8 to 38.9 ). We observed significant associations between the GPS and several anthropometric variables (such as BMI, body fat percentage, waist circumference and hip circumference; Table 1). The mean [95\% confidence interval (CI)] allele effect of the GPS was $+0.13[0.07-0.20] \mathrm{kg} / \mathrm{m}^{2} \quad\left(p=6.3 \times 10^{-5}\right)$ for BMI, $+0.14[0.05-0.24] \%(p=0.004)$ for body fat percentage, $+0.28[0.11-0.45] \mathrm{cm}(p=0.001)$ for waist circumference and $+0.24[0.11-0.37] \mathrm{cm}\left(p=3.7 \times 10^{-4}\right)$ for hip circumference. We did not detect a statistically significant association between the GPS and WHR.

Similar results were obtained after taking into account missing genotypes (Additional file 1: Table S4). Associations between the GPS and the waist and hip circumferences disappeared after further adjustment for BMI.

We also investigated the possible effect of interactions between the GPS and gender, physical activity (PA), smoking status and alcohol consumption on anthropometric variables but did not detect any significant interactions (data not shown).

To distinguish between the effects of the GPS and the effects of the covariables classically associated with BMI (age, gender, PA, smoking status and alcohol consumption), we compared the crude and adjusted models (Table 2). The GPS alone accounted for $1 \%$ of the variance in the BMI, whereas the covariables accounted for $6 \%$. Overall, the GPS and the covariables explained $7 \%$ of the variance in the BMI.

We also investigated the association between the GPS and the obesity risk. Each additional BMI-raising allele was associated with a $3 \%$ increase in the obesity risk $(\mathrm{OR}[95 \% \mathrm{CI}]=1.03$ [1.00-1.07]; $p=0.047)$.

The genetic predisposition score, glucose-related traits and the type 2 diabetes risk

Given that obesity is an important determinant of glycaemic traits and insulin resistance, we assessed the association between the GPS on one hand and fasting plasma glucose, HbA1c and insulin levels, the HOMA-IR and HOMA-B scores and the risk of T2D on the other. We

Table 1 Effect of the genetic predisposition score on anthropometric variables in the MONA LISA Lille study ( $n=1546)$

\begin{tabular}{lcccccc}
\hline Parameter & $\boldsymbol{\beta}$ & SE & LCL & UCL & $\boldsymbol{p}^{\mathbf{1}}$ & $\boldsymbol{p}^{\mathbf{2}}$ \\
\hline BMI $\left(\mathrm{kg} / \mathrm{m}^{2}\right)$ & 0.13 & 0.03 & 0.07 & 0.20 & $6.3 \times 10^{-5}$ & - \\
Body fat $(\%)$ & 0.14 & 0.05 & 0.05 & 0.24 & 0.004 & - \\
Waist $(\mathrm{cm})$ & 0.28 & 0.09 & 0.11 & 0.45 & 0.001 & 0.65 \\
Hip $(\mathrm{cm})$ & 0.24 & 0.07 & 0.11 & 0.37 & $3.7 \times 10^{-4}$ & 0.74 \\
Waist-to-hip ratio & 0.0006 & 0.0004 & -0.0003 & 0.0015 & 0.19 & 0.41 \\
\hline
\end{tabular}

The $\beta$ coefficients represent the effect sizes. SE: standard error. LCL: lower confidence limit; UCL: upper confidence limit.

${ }^{1} p$ values were adjusted for age, gender, physical activity, smoking status and alcohol consumption. ${ }^{2} p$ values were adjusted for age, gender, physical activity, smoking status, alcohol consumption and BMI. 
Table 2 Effects of the crude and adjusted genetic predisposition score on BMI in the MONA LISA Lille study ( $n=1546)$

\begin{tabular}{ccccccc}
\hline Models & $\boldsymbol{\beta}$ & SE & LCL & UCL & $\boldsymbol{p}$ & Explained variance (\%) \\
\hline Model 1 & 0.14 & 0.03 & 0.07 & 0.20 & $5.4 \times 10^{-5}$ & 1.0 \\
Model 2 & 0.13 & 0.03 & 0.07 & 0.2 & $6.3 \times 10^{-5}$ & 7.0 \\
\hline
\end{tabular}

The $\beta$ coefficients represent the effect sizes. SE: standard error. LCL: lower confidence limit; UCL: upper confidence limit.

Model 1: crude $p$ value. Model 2: $p$ value adjusted for age, gender, physical activity, smoking status and alcohol consumption.

detected significant associations between the GPS and higher fasting plasma glucose $(\beta \pm \mathrm{SE}=+0.017 \pm$ $0.008 \mathrm{mmol} / \mathrm{L}, \quad p=0.04)$, insulin $(\beta \pm \mathrm{SE}=+0.14 \pm 0.06$ $\mu \mathrm{IU} / \mathrm{mL}, p=0.008)$ and HbA1c levels $(\beta \pm \mathrm{SE}=+0.012 \pm$ $0.005 \%, p=0.01)$ and a higher HOMA-IR $(\beta \pm \mathrm{SE}=+0.06 \pm$ $0.02, p=0.0003$ ) (Table 3). The GPS was also significantly associated with a higher risk of T2D (adjusted OR [95\% $\mathrm{CI}]=1.06[1.00-1.11], p=0.03)$. However, these associations were no longer statistically significant after adjustment for BMI.

\section{Discussion}

Although the MONA LISA Lille study's statistical power was too low (68\%) to detect significant individual associations, 25 of the 31 investigated SNPs presented effects with the expected direction. Moreover, the effect alleles for the FTO rs9939609 and FANCL rs887912 SNPs were nominally associated with higher BMI. The GPS (corresponding to the cumulative contribution of the 31 validated BMI-associated SNPs) showed a significant, positive association with BMI. Each additional effect allele was associated with a mean increment of $0.13 \mathrm{~kg} / \mathrm{m}^{2}$ in the BMI (which corresponds to a weight increment of $376 \mathrm{~g}$ for a person measuring $1.70 \mathrm{~m}$ in height) and a $3 \%$ increase in the risk of obesity. The GPS was also significantly associated with body fat percentage and waist and hip circumferences, although the last two associations did not resist adjustment for BMI (suggesting that they were driven by overall general adiposity). The genetic susceptibility associated with the GPS explained only $1 \%$ of the variance in the BMI, whereas the combined effect of known lifestyle factors accounted for 6\%. Although it is clear that (i) genetic factors account for $40-70 \%$ of the population variation in BMI and (ii) the 31 SNPs studied here have been robustly validated as BMI-susceptible variants in GWASs and replication studies, the SNPs' combined effect on BMI and the obesity risk was quite small. However, our results are in agreement with previous reports $[8,10,11]$.

Gene-environment interactions may also account for variance in the BMI. Several studies have reported that PA is associated with a reduction in the GPS's impact on BMI $[12,13]$. Like others [12], we failed to detect significant interactions between the GPS and PA when considering several anthropometric traits (BMI, body fat percentage, waist and hip circumferences and WHR). Our failure to detect this interaction is probably due to the relatively small sample size. In fact, very large sample sizes are needed when exploring this type of interaction. For example, Ahmad et al. showed that a population size of 20,000 is required to detect a $\beta_{\mathrm{GE}}$ interaction effect of $-0.07 \mathrm{~kg} / \mathrm{m}^{2}$ [13].

Given that obesity is a major risk factor for insulin resistance [14], the accumulation of obesity risk alleles may alter glucose metabolism and predispose the individual to T2D. To evaluate this hypothesis, we looked at whether the GPS was associated with glucose-related variables and the T2D risk in the MONA LISA Lille study. Indeed, we found significant associations between the GPS on one hand and higher fasting plasma glucose, insulin and HbA1c levels and insulin resistance on the other. We also showed that each additional BMI-raising allele was associated with a $6 \%$ increment in the T2D risk. Our results in a general population sample are consistent with previous reports. In a French case-control study, each additional allele in the GPS was associated with higher insulin resistance and a 3\% increase in the T2D risk [15]. In the EPIC prospective cohort study, each additional allele in the GPS was also associated with a $4 \%$ increase in the T2D risk [10]. In both these previous studies (as in the present study), all the statistically significant associations were abolished after adjustment for BMI - meaning that overall general adiposity explained the association between the GPS and insulin resistance or $\mathrm{T} 2 \mathrm{D}$.

Table 3 Associations between the genetic predisposition score and glucose-related variables in the MONA LISA Lille study

\begin{tabular}{|c|c|c|c|c|c|c|}
\hline & $\beta$ & SE & $\mathrm{LCL}$ & UCL & $\begin{array}{c}\text { Model } 1 \\
p\end{array}$ & $\begin{array}{c}\text { Model } 2 \\
p\end{array}$ \\
\hline Fasting glucose $(\mathrm{mmol} / \mathrm{L})$ & 0.017 & 0.008 & 0.001 & 0.033 & 0.04 & 0.35 \\
\hline Fasting insulin ( $\mu \mathrm{lU} / \mathrm{mL})$ & 0.14 & 0.06 & 0.03 & 0.24 & 0.008 & 0.46 \\
\hline $\mathrm{HbA1c}(\%)$ & 0.012 & 0.005 & 0.003 & 0.021 & 0.01 & 0.10 \\
\hline HOMA-IR & 0.06 & 0.02 & 0.03 & 0.10 & 0.0003 & 0.10 \\
\hline HOMA-B & 1.17 & 0.63 & -0.07 & 2.42 & 0.18 & 0.83 \\
\hline
\end{tabular}

The $\beta$ coefficients represent the effect sizes. SE: standard error. LCL: lower confidence limit; UCL: upper confidence limit.

Model 1: values were adjusted for age, gender, physical activity, smoking status and alcohol consumption.

Model 2: values were adjusted for age, gender, physical activity, smoking status, alcohol consumption and BMI. 


\section{Conclusions}

Our results showed that the combination of common genetic variants was moderately associated with BMI and BMI-related variables in a sample of the general population from northern France. Despite the fact that the heritability of BMI is estimated to be $40-70 \%$ [5], the combination of 31 validated, $\mathrm{BMI}$-associated loci only explained only $1 \%$ of the variance in the BMI (i.e. less than $2-4 \%$ of the heritability) [8]. Hence, characterization of this unexplained heritability requires other approaches.

\section{Methods}

\section{The MONA LISA Lille study}

The MONA LISA (Monitoring National du Risque Artériel; National Monitoring of Arterial Risk) Lille study was a population-based, cross-sectional study of a representative sample of 1578 participants recruited from within the Lille urban area in northern France. In accordance with the French legislation on biomedical research, the study protocol was approved by the appropriate independent ethics committee (Comité Consultatif de Protection des Personnes dans la Recherche Biomédicale de Lille) and written informed consent was obtained from all participants. The study design and methods are described in the Additional file 1: methods. Briefly, anthropometric traits were recorded during a physical examination of each individual and a blood sample was collected (for DNA extraction and clinical biochemistry assays). The BMI was calculated according to the Quetelet equation. Obesity was defined as a BMI of $30 \mathrm{~kg} / \mathrm{m}^{2}$ or more. Type 2 diabetes was defined according to the 1997 American Diabetes Association definition (fasting plasma glucose $\geq 7.0 \mathrm{mmol} / \mathrm{l}$ and/or treatment for diabetes, including diet and/or oral antidiabetic drugs and/or insulin) [16].

\section{Genotyping}

Single nucleotide polymorphisms were genotyped using KASPar technology (KBioscience, Hoddesdon, UK). The genotyping success rates ranged from $98.1 \%$ to $99.6 \%$.

\section{Statistical analysis}

Statistical analyses were performed with SAS 9.1 software (SAS Institute Inc., Cary, NC, USA).

The Hardy-Weinberg equilibrium was tested using a $x^{2}$ test with one degree of freedom.

The GPS was derived as described previously [17]. Briefly, a weighting method was used to calculate the GPS on the basis of 31 SNPs. Each SNP was weighted according to its relative effect size (i.e. the $\beta$ coefficient). In order to measure the effect of each SNP on BMI with greater accuracy and precision, $\beta$ coefficients were derived as described by Speliotes et al. [8]. We rescaled the weighted scores to reflect the number of risk alleles.
Hence, each point on the GPS corresponded to one risk allele. When calculating the GPS, missing genotype data were replaced with the average allele count for the corresponding SNPs. However, individuals with missing genotypes for more than $10 \%$ of the loci were excluded from the GPS analyses $(n=30)$.

We used general linear regression models to test the associations of individual BMI-related SNPs and the GPS with adiposity-related traits (including BMI, body fat percentage, WHR, waist circumference and hip circumference) and glucose-related traits (assuming an additive effect of the BMI-increasing alleles). A logistic regression model was used to test the association between the GPS and the risk of obesity or T2D. Interactions between the GPS on one hand and gender, PA, smoking status and alcohol consumption on the other were tested by including the GPS, interaction variables and the interaction terms (GPS $\mathrm{x}$ interaction variables) in general linear regression models.

The associations between genetic variants and BMI, obesity and interactions were adjusted for age, gender, smoking status, PA and alcohol consumption. The associations between genetic variants and body fat percentage, WHR, waist circumference and hip circumference were adjusted for age, gender, smoking status, PA, and alcohol consumption including or not BMI, depending of models. The associations between genetic variants and biological parameters and the T2D risk were adjusted for age, gender, BMI, smoking status, PA and alcohol consumption. Data distributions for plasma glucose and insulin levels and HOMA-IR and HOMA-B scores were normalized by log transformation.

Bonferroni correction was used to adjust for the Hardy-Weinberg equilibrium and for the multiple testing in the individual obesity-related trait analyses. The threshold for statistical significance was set to $p \leq 0.0016$ (for 31 independent SNPs). Nominal significance was defined as $0.0016<p<0.05$.

For the GPS analyses, the threshold for statistical significance was set to $p \leq 0.05$.

The power calculations for association analyses (performed a priori using Quanto v1.2.4 software (http:// biostats.usc.edu/Quanto.html) on the basis of the mean BMI values from the MONA LISA Lille study and the effect allele frequencies and effect sizes originally reported by Speliotes et al. [8]) indicated that the statistical power of our study (for detecting a significant association between an individual SNP and BMI with a one-sided $p$ value of 0.05 ) was $68 \%$.

The power calculations for the GPS analysis were performed using the pwr package developed by Stéphane Champely. The statistical power for detecting significant association between GPS and BMI (using a $p$ value at $0.05)$ was $99 \%$. 


\section{Additional file}

Additional file 1: Table S1. Characteristics of the participants in the MONA LISA Lille study $(n=1578)$. Table S2. Genotype and allele distributions of the 31 successfully genotyped SNPs in the MONA LISA Lille study. Table S3. Associations between the 31 SNPs and the anthropometric variables in the MONA LISA Lille study $(n=1578)$. Table S4. Effect of the GPS on anthropometric variables in the MONA LISA Lille study for fully genotyped participants $(n=1326)$. Methods. The MONA LISA Lille study.

\section{Abbreviations}

BMI: Body mass index; Cl: Confidence interval; GPS: Genetic predisposition score; GWAS: Genome-wide association study; OR: Odds ratio; PA: Physical activity; SE: Standard error; SNP: Single nucleotide polymorphism; T2D: Type 2 diabetes; WHR: Waist-to-hip ratio; WHO: World health organization.

\section{Competing interests}

The authors declare that they have no competing interests.

\section{Authors' contributions}

DC, JD, PA, LG and AM designed the study and supervised the project. DC, JD and PA participated in the recruitment of participants. LG performed the statistical analyses. LG and AM interpreted the results. LG wrote the manuscript. LG and AM had primary responsibility for final content. All authors read and approved the final manuscript.

\section{Acknowledgments}

The MONA LISA Study was made possible by an unrestricted grant from Pfizer and a grant from the French Agence Nationale de la Recherche (ANR-05-PNRA-018).

Received: 14 January 2014 Accepted: 19 May 2014

Published: 23 May 2014

\section{References}

1. Rabin BA, Boehmer TK, Brownson RC: Cross-national comparison of environmental and policy correlates of obesity in Europe. Eur J Public Health 2007, 17:53-61.

2. Finucane MM, Stevens GA, Cowan MJ, Danaei G, Lin JK, Paciorek CJ, Singh GM, Gutierrez HR, Lu Y, Bahalim AN, Farzadfar F, Riley LM, Ezzati M: National, regional, and global trends in body-mass index since 1980: systematic analysis of health examination surveys and epidemiological studies with 960 country-years and 9.1 million participants. Lancet 2011, 377:557-567.

3. Kopelman P: Health risks associated with overweight and obesity. Obes Rev 2007, 8(1):13-17.

4. WHO: WHO. In World Health Organisation; 2011. Available at: http://www. who.int/topics/obesity/en/.

5. Maes HH, Neale MC, Eaves $\amalg$ : Genetic and environmental factors in relative body weight and human adiposity. Behav Genet 1997, 27:325-351.

6. Atwood LD, Heard-Costa NL, Cupples LA, Jaquish CE, Wilson PW, D'Agostino RB: Genomewide linkage analysis of body mass index across 28 years of the Framingham Heart Study. Am J Hum Genet 2002, 71:1044-1050.

7. Blakemore Al, Froguel P: Is obesity our genetic legacy? J Clin Endocrinol Metab 2008, 93:S51-S56.

8. Speliotes EK, Willer CJ, Berndt SI, Monda KL, Thorleifsson G, Jackson AU, Lango AH, Lindgren CM, Luan J, Magi R, Randall JC, Vedantam S, Winkler TW, Qi L, Workalemahu T, Heid IM, Steinthorsdottir V, Stringham HM, Weedon MN, Wheeler E, Wood AR, Ferreira T, Weyant RJ, Segre AV, Estrada K, Liang L, Nemesh J, Park JH, Gustafsson S, Kilpelainen TO, et al: Association analyses of 249,796 individuals reveal 18 new loci associated with body mass index. Nat Genet 2010, 42:937-948.

9. Vliet-Ostaptchouk JV, den Hoed M, Luan J, Zhao JH, Ong KK, van der Most PJ, Wong A, Hardy R, Kuh D, van der Klauw MM, Bruinenberg M, Khaw KT, Wolffenbuttel BH, Wareham NJ, Snieder H, Loos RJ: Pleiotropic effects of obesity-susceptibility loci on metabolic traits: a meta-analysis of up to 37,874 individuals. Diabetologia 2013, 56:2134-2146.
10. Li S, Zhao JH, Luan J, Langenberg C, Luben RN, Khaw KT, Wareham NJ, Loos RJ: Genetic predisposition to obesity leads to increased risk of type 2 diabetes. Diabetologia 2011, 54:776-782.

11. Li S, Zhao JH, Luan J, Luben RN, Rodwell SA, Khaw KT, Ong KK, Wareham NJ, Loos RJ: Cumulative effects and predictive value of common obesitysusceptibility variants identified by genome-wide association studies. Am J Clin Nutr 2010, 91:184-190.

12. Jaaskelainen T, Paananen J, Lindstrom J, Eriksson JG, Tuomilehto J, Uusitupa M: Genetic predisposition to obesity and lifestyle factors - the combined analyses of twenty-six known BMI- and fourteen known waist: hip ratio (WHR)-associated variants in the Finnish Diabetes Prevention Study. Br J Nutr 2013, 110:1856-1865.

13. Ahmad S, Rukh G, Varga TV, Ali A, Kurbasic A, Shungin D, Ericson U, Koivula RW Chu AY, Rose LM, Ganna A, Qi Q, Stancakova A, Sandholt CH, Elks CE, Curhan G, Jensen MK, Tamimi RM, Allin KH, Jorgensen T, Brage S, Langenberg C, Aadahl M, Grarup N, Linneberg A, Pare G, Magnusson PK, Pedersen NL, Boehnke M, Hamsten A, et al: Gene x physical activity interactions in obesity: combined analysis of 111,421 individuals of European ancestry. PLoS Genet 2013, 9:e1003607.

14. Kahn SE, Hull RL, Utzschneider KM: Mechanisms linking obesity to insulin resistance and type 2 diabetes. Nature 2006, 444:840-846.

15. Robiou-du-Pont S, Bonnefond A, Yengo L, Vaillant E, Lobbens S, Durand E, Weill J, Lantieri O, Balkau B, Charpentier G, Marre M, Froguel P, Meyre D: Contribution of 24 obesity-associated genetic variants to insulin resistance, pancreatic beta-cell function and type 2 diabetes risk in the French population. Int J Obes (Lond) 2013, 37:980-985.

16. Expert Committee on the Diagnosis and Classification of Diabetes Mellitus: Report of the expert committee on the diagnosis and classification of diabetes mellitus. Diabetes Care 2003, 26(1):S5-S20. S5-20.

17. Qi Q, Chu AY, Kang JH, Jensen MK, Curhan GC, Pasquale LR, Ridker PM, Hunter DJ, Willett WC, Rimm EB, Chasman DI, Hu FB, Qi L: Sugarsweetened beverages and genetic risk of obesity. N Engl J Med 2012, 367:1387-1396.

doi:10.1186/1471-2156-15-62

Cite this article as: Goumidi et al:: Effects of established BMI-associated loci on obesity-related traits in a French representative population sample. BMC Genetics 2014 15:62.

\section{Submit your next manuscript to BioMed Central and take full advantage of:}

- Convenient online submission

- Thorough peer review

- No space constraints or color figure charges

- Immediate publication on acceptance

- Inclusion in PubMed, CAS, Scopus and Google Scholar

- Research which is freely available for redistribution 